
3
Research Square
Preprints are preliminary reports that have not undergone peer review.
They should not be considered conclusive, used to inform clinical practice, or referenced by the media as validated information.

\title{
M2 macrophage-secreted exosomes promote metastasis and increase vascular permeability in hepatocellular carcinoma
}

\section{Yiwei Lu (D1029575136@qq.com)}

Nanjing Medical University https://orcid.org/0000-0003-2603-2302

\section{Guoyong Han}

Nanjing Medical University

\section{Yao Zhang}

Nanjing Medical University

Long Zhang

Nanjing Medical University

\section{Zhi Li}

Nanjing Medical University

\section{Qingyuan Wang}

Nanjing Medical University

Zhiqiang Chen

Nanjing Medical University

Xuehao Wang

Nanjing Medical University

Jindao Wu

Nanjing Medical University

\section{Research Article}

Keywords: M2 macrophages, exosomes, EMT, angiogenesis, vascular permeability, miR-23a-3p

Posted Date: January 24th, 2022

DOI: https://doi.org/10.21203/rs.3.rs-1225380/v1

License: (c) (1) This work is licensed under a Creative Commons Attribution 4.0 International License. Read Full License 


\section{Abstract}

Background: Metastasis is the main feature of malignant tumors and is mainly responsible for its high mortality, particularly in hepatocellular carcinoma (HCC). Therefore, it is important to explore the mechanism of tumor metastasis. Recently, tumor-associated macrophages (TAMs) have been shown to promote tumor progression, whereas TAM-derived molecules that are involved in HCC metastasis require further investigation.

Methods: THP-1 were treated with IL-4 (Interleukin-4) and IL-13 (Interleukin-13) for M2 polarized macrophages. Exosomes derived from M2 macrophages were characterized. HCC cells or human umbilical vein endothelial cells (HUVEC) were cocultured with M2 macrophages or treated with M2 macrophage-secreted exosomes. Transwell ${ }^{\circledR}$, tube formation, and endothelial permeability assays were performed. RT-PCR, western-blotting, immunofluorescence and ELISA were used to assess mRNA and protein expression levels. MiRNA expression profiles of exosomes derived from M2 and M0 macrophages were analyzed.

Results: M2 macrophage infiltration is correlated to metastasis and poor prognosis of HCC patients. M2derived exosomes were absorbed by HCC and HUVEC cells and promoted epithelial-mesenchymal transition (EMT), vascular permeability, and angiogenesis. MiR-23a-3p was significantly higher in M2derived exosomes and hnRNPA1-mediated miR-23a-3p packaging into exosomes. Phosphatase and tensin homolog (PTEN) and tight junction protein 1 (TJP1) were the targets of miR-92a-3p, as confirmed by luciferase reporter assays. HCC cells cocultured with M2-derived exosomes secreted more GM-CSF, VEGF, G-CSF, MCP-1 and IL-4, which in turn recruited more M2 macrophages.

Conclusions: Our findings suggest that M2 macrophage-derived miR-23a-3p enhances HCC metastasis by promoting EMT and angiogenesis, as well as increasing vascular permeability.

\section{Background}

Hepatocellular carcinoma (HCC) is one of the most common malignant tumors, with about 800,000 new cases diagnosed each year. It has a high mortality rate of about 700,000 deaths each year ${ }^{1,2}$. For early HCC, surgical resection or liver transplantation can achieve a good prognosis, but some patients lose surgical opportunities when intrahepatic or distant metastases occurs. Metastasis is one of the main causes of cancer-related deaths in $\mathrm{HCC}^{3,4}$. Therefore, exploring the mechanism of metastasis is of important clinical significance for improving the survival of individuals with advanced HCC.

The tumor microenvironment is composed of tumor cells, immune cells, interstitial cells, and secreted active mediators ${ }^{5,6}$. In the tumor microenvironment, the interactions among various types of cells reshape each other and together maintain the occurrence and development of tumors. Among the many mutant cells infiltrated in the tumor environment, tumor-associated macrophages (TAM) have the largest number, accounting for $30-65 \%$ in $\mathrm{HCC}^{7-9}$. Macrophages can be activated in various microenvironments with 
different properties and have a wide range of activated phenotypes and functions. M2 macrophages highly express arginase 1 (Arg1), macrophage mannose receptor (MMR), and scavenger receptor ${ }^{10,11}$. Studies have found that TAMs promote tumor progression through immunosuppression. In HCC, TAMs secrete more transforming growth factor beta 1 (TGF-beta 1) and induce HCC cells to obtain stemness ${ }^{12}$.

Exosomes are a kind of 50-100-nm lipid bilayer microvesicles that are secreted by the body cells under normal and pathological conditions. Exosomes can encapsulate proteins, nucleic acids, and lipids to transport substances between cells, thereby affecting the function of recipient cells ${ }^{13}$. Studies has shown that exosomes play an important role in tumors. In a hypoxic environment, tumor cells secrete exosomes with miR-301a and activate the PTEN/PI3K signaling pathway to induce M2 polarization of macrophages that promote pancreatic cancer metastasis ${ }^{14}$. The acidic microenvironment in HCC induces higher expression of miR-21 and miR-10b in tumor cells and promotes cancer cell proliferation and metastasis ${ }^{15}$. However, whether M2 macrophage-derived exosomes promote HCC metastasis requires further investigation.

This study aimed to investigate the effect of M2 macrophage exosomes on HCC cell metastasis. We found that M2 macrophage infiltration was significantly higher in HCC tissues with metastasis compared with non-metastasis and is negatively correlated with prognosis. M2 macrophage exosomes promote HCC metastasis by transmitting miR-23a-3p into HCC cells and HUVESs to induce EMT and angiogenesis, as well as increase vascular permeability. Our study provides a new theoretical basis for the treatment of HCC metastasis.

\section{Materials And Methods Cell lines and culture}

The HCC cell lines Huh-7 and SMMC-7721 were used in this study. The cell lines were provided and identified by our laboratory of living donor liver transplantation. The cells were cultured in Dulbecco's modified Eagle medium (DMEM; Gibco, Grand Island, NY, USA) containing 10\% fetal bovine serum (Gibco), $100 \mathrm{U} / \mathrm{mL}$ penicillin, and $100 \mu \mathrm{g} / \mathrm{mL}$ streptomycin (Invitrogen, Carlsbad, CA, USA) at $37^{\circ} \mathrm{C}$ in $5 \%$ C02. RPMI-1640 (DMEM; Gibco) containing 10\% fetal bovine serum, $100 \mathrm{U} / \mathrm{mL}$ penicillin, and $100 \mu \mathrm{g} / \mathrm{mL}$ streptomycin was used for THP-1 cells. For M2 polarized macrophages, THP-1 cells were treated with 5 $\mathrm{ng} / \mathrm{mL}$ PMA (Sigma, USA) for $24 \mathrm{~h}$ and then incubated with $20 \mathrm{ng} / \mathrm{mL} \mathrm{IL}-4$ plus $20 \mathrm{ng} / \mathrm{mL} \mathrm{IL}-13$ (PeproTech, USA) for 48 h. M2 polarized macrophages were identified by microscopy, flow cytometry, and immunofluorescence.

\section{Isolation of macrophage exosomes}

M2 macrophages obtained from THP-1 were incubated with 10\% exosome-free FBS for $48 \mathrm{~h}$. Then, the medium was collected and centrifuged at $1,000 \mathrm{~g}$ for $5 \mathrm{~min}$ at room temperature and then at $15,000 \mathrm{~g}$ for 
15 min at $4^{\circ} \mathrm{C}$, followed by filtration with $0.22-\mu \mathrm{m}$ membrane to remove cells, cell fragments, and organelles. The supernatant was then centrifuged at $110,000 \mathrm{~g}$ for $70 \mathrm{~min}$ at $4^{\circ} \mathrm{C}$. The precipitate was then collected and resuspended in PBS. The exosomes were characterized by transmission electron microscopy and western blotting.

\section{Transmission electron microscopy (TEM)}

For TEM, the exosomes were resuspended in PBS, dropped onto a copper mesh, and stained with $1 \%$ uranyl acetate. The mesh was dried for $5 \mathrm{~min}$ and observed under a transmission electron microscope (FEl, Tecnai G2 spirit).

\section{Exosome tracking}

The extracted exosomes were incubated with $0.5 \mathrm{~mL}$ of diluent C. Approximately $4 \mathrm{UL}$ of a PKH67 dye solution were incubated with $0.5 \mathrm{~mL}$ of diluent $\mathrm{C}$. The exosomes and PKH67 were mixed and incubated overnight at $4^{\circ} \mathrm{C}$. The mixture was rinsed with PBS to remove excess dyes. The prepared dye solution was seeded to the prepared cells for $24 \mathrm{~h}$ of incubation. Then, images of the cells were captured under a laser confocal microscope.

\section{Cell transfection}

For the lentivirus (Genepharma, Shanghai, China), cells were plated into six-well plates at a density of $2 \times 105$ cells per well. When the density reached $30-40 \%$ after incubation, the lentiviruses were transfected into the HCC cells. When cell confluency reached $80-90 \%$, the cells were transferred into $100-\mathrm{mm}$ dishes and selected for 2 weeks using puromycin $(10 \mu \mathrm{g} / \mathrm{mL})$. For shRNA, Lipofectamine 3000 (Invitrogen) was used according to the manufacturer's protocol. Briefly, the cells were plated in six-well plates at a density of $1.5 \times 105$ cells per well one day before transfection. When the cells were in the log phase, a mimic was added to each well. The medium was replaced after incubation for $6 \mathrm{~h}$. The cells were harvested $48-72 \mathrm{~h}$ later for analysis.

\section{RNA isolation and quantitative real-time PCR}

Total RNA was extracted from human specimens and cells using TRIzol reagent (Takara, Dalian, China) according to the manufacturer's instructions. RNA quality and concentration were assessed using a Nanodrop 2000 system (NanoDrop Technologies, IL, USA). The cDNAs of miRNA were reverse transcribed using the reverse translate kit PrimeScript RT Master Mix (DRR037A; Takara) according to the manufacturer's protocol. Sequence-specific primers for U6 and miR-23a-3p were synthesized by RiboBio (Guangzhou, China). Real-time polymerase chain reaction was performed with SYBR Premix ExTaq (TaKaRa, Dalian, China) with the ABI Prism 7900HT (Applied Biosystems, Foster City, CA, USA). 
Glyceraldehyde-3-phosphate dehydrogenase (GAPDH) was used as internal control for mRNA quantification. The relative expression was calculated using the 2- $\Delta \Delta C T$ method. The primer sequences used in real-time PCR were displayed in Supplementary Table S1.

\section{Western blotting}

Cells and tissue specimens were lysed using a lysis buffer (10 mM Tris, 1 mM EDTA, 1 mM DTT, 60 mM $\mathrm{KCl}, 0.5 \% \mathrm{v} / \mathrm{v} \mathrm{NP}-40$, and protease inhibitors) on ice. Protein samples $(20 \mu \mathrm{g})$ were separated using $10 \%$ sodium dodecyl sulfate-polyacrylamide gel electrophoresis (SDS-PAGE) and transferred onto a polyvinylidene difluoride membrane. After blocking, the bands were incubated with specific antibodies. Proteins were visualized with an enhanced chemiluminescence detection kit according to the manufacturer's recommendations (Beyotime, Shanghai, China). Relative protein levels were calculated based on GAPDH levels. All antibodies used in the study was displayed in Supplementary Table S2

\section{Immunohistochemistry}

A diaminobenzidine detection kit (Maixin-Bio, Fuzhou, China) was used for immunohistochemical staining according to the manufacturer's instructions. The dissected HCC and peritumor tissues were fixed and embedded in paraffin. Then, $5-\mu \mathrm{m}$ thick consecutive sections were cut and mounted on glass slides, dewaxed, rehydrated, and antigen retrieved. The sections were incubated with a primary antibody at $37^{\circ} \mathrm{C}$ for $1 \mathrm{~h}$, a biotin-labeled secondary antibody for $10 \mathrm{~min}$, and a streptavidin-peroxidase conjugate for $10 \mathrm{~min}$. A $0.02 \%$ diaminobenzidine solution was used as chromogen to visualize peroxidase activity. The sections were lightly counterstained with hematoxylin, mounted with Permount, and examined with light microscopy.

\section{Transwell ${ }_{\circledast}$}

Migration and invasion analysis was performed using Transwell囚 chambers (Corning Incorporated, Corning, NY, USA). For migration, following suspension in serum-free medium, $3 \times 104$ cells were seeded into the Transwell ${ }^{\circledR}$ insert supplemented with DMEM containing $5 \%$ serum. After $48 \mathrm{~h}$ of incubation, the cells on the bottom side of the membrane were fixed with $95 \%$ alcohol and stained with crystal violet for $20 \mathrm{~min}$ at room temperature. Then, the number of cells on the lower side of the filter was counted under a microscope. Each experiment was performed in triplicate. For HUVEC cell migration, $1 \times 104$ cells were seeded and then incubated $24 \mathrm{~h}$. For invasion, Matrigel ${ }^{\circledR}$ was plated in the upper surface before cell inoculation. The other steps were the same as in the migration assay.

\section{Endothelial permeability assay in vitro}


Endothelial permeability assay was performed according to a previous study ${ }^{16}$. Briefly, $2 \times 104$ of HUVEC cells were seeded into 24 -well Transwell $\circledast$ filters (8- $\mu \mathrm{m}$ pore size; Corning) and then incubated when cell confluency reached $100 \%$. Then, the 24 -well Transwell ${ }^{\circledR}$ filters were carefully washed with PBS. Then, 20 $\mathrm{mg} / \mathrm{mL}$ rhodamine-dextran (average MW: 70,000; Sigma-Aldrich) was added into the upper chamber and after $1 \mathrm{~h}$ of incubation, the lower medium was collected and assessed under laser confocal scanning microscopy.

\section{Transendothelial invasion assay}

A transendothelial invasion assay was performed according to a previous study ${ }^{17}$. Endothelial monolayers were prepared as earlier described. The 24-well Transwell $\circledast$ filters were placed into new 24well plates, and then $5 \times 104$ of GFP-positive HCC cells were seeded into the upper chamber, and the lower chamber was filled with $750 \mu \mathrm{L}$ of $10 \%$ exosome-depleted FBS-SFM. After a 12-h incubation, the cells in the upper and lower chambers were collected and counted under a fluorescent microscope.

\section{Tube formation assay}

Growth factor-reduced Matrigel ${ }^{\circledR}$ (BD Biosciences, San Jose, CA, USA) was diluted with serum-free medium at a 1:1 ratio and then $300 \mu \mathrm{L}$ were placed into each well of 24-well plates. The plates were incubated for $30 \mathrm{~min}$. The different group of HUVEC cells were suspended at a density of $1.2 \times 106$, and $100 \mu \mathrm{L}$ of the cell suspensions were seeded into the 24 -well plates. After $4-6 \mathrm{~h}$ of incubation, tube images were captured using a digital camera. Tube formation was determined using ImageJ software.

\section{Immunofluorescence assay}

For immunofluorescence, cells were fixed with $4 \%$ formaldehyde, permeabilized with $0.2 \%$ Triton X-100, and blocked with $1 \%$ bovine serum albumin in PBS. Cells were incubated with the primary antibody overnight at $4^{\circ} \mathrm{C}$. The cells were then incubated with FITC-conjugated goat anti-rabbit IgG after three washes. Finally, the cells were washed and mounted with a mounting medium containing 4,6-diamidino2-phenylindole (DAPI). Images were captured with a scanning microscope.

\section{ELISA}

Briefly, the cell culture supernatant was collected, centrifuged at $1,000 \mathrm{~g}$ at $4^{\circ} \mathrm{C}$ for $30 \mathrm{~min}$, and the supernatant was stored at $-80^{\circ} \mathrm{C}$ until use. Samples to be tested were thawed on ice. Standard wells were set up, and then $50 \mu \mathrm{L}$ of pre-replaced standards at different concentrations were added to each well. Then, $50 \mu \mathrm{L}$ of each sample to be tested were added into each well. Later, $50 \mu \mathrm{L}$ of horseradish were added per well and incubated for $1 \mathrm{~h}$ at $37^{\circ} \mathrm{C}$. The liquid was later discarded and then $300 \mu \mathrm{L}$ of cleaning solution were added. After 2 min, the cleaning solution was discarded, and the microwell plate was patted 
dry on the absorbent paper to remove any residual liquid. The microwell plate was rinsed for another 5 times, and then $50 \mu \mathrm{L}$ of the substrate were added to each well and incubated for $15 \mathrm{~min}$. Then, $50 \mu \mathrm{L}$ of a stop solution was added to terminate the reaction, and then the absorbance of each well was assessed at a wavelength of $450 \mathrm{~nm}$.

\section{CCK-8 assay}

Briefly, cells were plated into 96 -well plates at a density of 3,000 cells/well. In each well, $10 \mu \mathrm{L}$ of CCK-8 were added at various time points $(0,24,48$, and $72 \mathrm{~h})$, and the cells were incubated at $37^{\circ} \mathrm{C}$ for another 2 $\mathrm{h}$. The absorbance at a wavelength of $450 \mathrm{~nm}$ was measured and used to generate cell growth curves.

\section{RIP assay}

RIP assays were performed using an RIP RNA-binding protein immunoprecipitation kit (Millipore, USA) according to the instruction. Briefly, the cells were lysed on ice-cold lysates supplemented with protease inhibitors, RNase inhibitors, and $1 \mathrm{mM} \mathrm{PMSF}$ and centrifuged at $1,500 \mathrm{rpm}$ at $4^{\circ} \mathrm{C}$ for $15 \mathrm{~min}$. The protein extract ( $1 \mathrm{mg}$ ) was incubated with $3 \mu \mathrm{g}$ of rabbit anti hnRNPA1 antibody (Cell Signaling Technology, USA) or rabbit IgG (Proteintech, USA) in reverse rotation at $4^{\circ} \mathrm{C}$ overnight. Then, about $30 \mu \mathrm{L} \mathrm{A/G}$ protein beads were added, and the cells were suspended and incubated at $4^{\circ} \mathrm{C}$ for $4 \mathrm{~h}$. Then, the beads were washed five times.Co-immunoprecipitated miRNA was extracted by Ambion. The separated RNA was analyzed.

\section{Luciferase assay}

MiRNA targets were predicted using the TargetScan and miRDB. Cells $(5 \times 104)$ were seeded into 24-well plates and cultured for $24 \mathrm{~h}$. The reporter luciferase plasmid (100 ng), pGL3, pGL3--mut, or control luciferase plasmid, and $5 \mathrm{ng}$ pRL-TK Renilla plasmid (Promega, Madison, WI, USA) was transfected into the cells using Lipofectamine 3000 according to the manufacturer's instructions. Luciferase and Renilla signals were measured $48 \mathrm{~h}$ after transfection using a Dual-Luciferase Reporter Assay Kit (Promega) according to the manufacturer's protocol.

\section{Statistical analyses}

Statistical analyses were performed using Prism 5.0 (GraphPad Software, La Jolla, CA, USA) software. The results are presented as the mean \pm standard error of the mean (SEM). The Student's t test was used for assessment of group differences. Differences with $\mathrm{P}<0.05$ were considered statistically significant.

\section{Results}

M2 macrophage infiltration is correlated with metastasis and poor prognosis in HCC. 
To study the relationship between M2 macrophage infiltration and HCC metastasis, the expression of CD163 (a marker of M2 macrophage) was assessed by immunohistochemistry in metastatic and nonmetastatic HCC tissues. The results show that CD163 expression was significantly higher in metastatic HCC tissues (Fig. 1a). In addition, M2 macrophage infiltration was negatively correlated with prognosis of HCC patients (Fig. 1b). To further explore M2 macrophage-associated pathways on HCC metastasis, we performed Gene Set Enrichment Analysis (GSEA). We found that EMT and angiogenesis were related to M2 macrophage infiltration (Fig. 1c). These results suggest that M2 macrophage infiltration is correlated with HCC metastasis and prognosis. The M2 macrophages might have influenced HCC metastasis via EMT and angiogenesis.

\section{M2 macrophage-derived exosomes promote HCC cell metastasis and EMT. Human THP-1 monocytes} were used to generate M2 macrophages. The polarized macrophages were confirmed by microscopy, flow cytometry, and immunofluorescence. The results showed that M2 macrophage were spindle-shaped and expressed by CD163 (Supporting Supplementary Fig. S1a-c). Exosomes are a component of cell signaling that play an important role in the tumor microenvironment. To further study whether exosomes participate in the regulation of HCC metastasis by macrophages, we collected macrophage exosomes. The exosomes were cup-shaped, 50-100 nm diameter structures, and expressed CD63, CD81, and CD9 (Fig. 2a-c). M2 macrophage exosomes could be absorbed by HCC cells when co-cultured, as confirmed by PKH67 fluorescence staining (Fig. 2d). Fig. 2e shows that when co-cultured with M2 macrophageconditioned medium, the migration and invasion ability of HCC cells were enhanced. M2 exosomes alone also promoted $\mathrm{HCC}$ metastasis. When the M2 macrophage-conditioned medium was treated with GW4869, which inhibits the release of exosomes, the enhanced migration and invasion ability of HCC in M2 macrophage-conditioned medium was reduced. These findings suggest that M2 macrophage-derived exosomes promote $\mathrm{HCC}$ cell metastasis. As previously reported, M2 macrophages are correlated with EMT in HCC tissues, and so we investigated whether M2 macrophage exosomes influence EMT of HCC cells. Fig. $2 \mathrm{f}$ shows that $\mathrm{M} 2$ macrophage exosomes reduce the expression of E-cadherin, whereas increase $\mathrm{N}$-cadherin and vimentin expression. These findings suggest that M2 macrophage exosomes promote metastasis and EMT of HCC cells.

\section{M2 macrophage-derived exosomes promote angiogenesis and increase vascular permeability.}

Angiogenesis and increased vascular permeability are important components of tumor metastasis. M2 macrophages were correlated with angiogenesis in HCC tissues, as earlier described. Thus, we further investigated the influence of M2 macrophage exosomes on angiogenesis. First, we found that M2 macrophage exosomes were internalized by HUVECs. M2 macrophage exosomes increased the secretion of VEGFA and proliferation and metastasis of HUVECs (Fig. 3a-d). Then, we performed a tube formation assay. Fig. 3e shows that M2 macrophage exosomes enhance angiogenesis. Intercellular adhesion plays an important role in maintaining endothelial integrity. Increased vascular permeability facilitates the penetration of tumor cells into blood vessels for distant metastasis. We found that M2 macrophage exosomes decreased the expression of TJP-1 and tight junction-related proteins occludin and claudin 5 , and increased vascular permeability, indicating the number of GFP-positive cells that invaded through 
HUVEC monolayers and amount of rhodamine B isothiocyanate-dextran (Fig. $3 f-h$ ). These findings show that M2 macrophage exosomes promote angiogenesis and increase vascular permeability.

\section{M2 macrophage-secreted miR-23a-3p promotes HCC metastasis.}

Exosomes contain a large number of proteins and miRNAs which are transfered to recipient cells. To investigate how M2 macrophage exosomes promote HCC metastasis, we performed a miRNA microarray assay. The results suggested that miR-23a-3p is upregulated in M2 macrophage exosomes, which was confirmed by RT-PCR (Fig. 4a). Meanwhile, Cy3-miR-23a-3p internalization by HCC cells and HUVECs was also evaluated by fluorescence microscopy (Fig. 4b). When HCC cells and HUVECs were cocultured with M2 macrophages, the expression of miR-23a-3p in HCC cells and HUVECs was upregulated. However, this upregulation was inhibited by GW4869, which inhibited the release of exosomes (Supporting Supplementary Fig. 2a). These findings suggest that M2 macrophages transferred miR-23a-3p through exosomes. To investigate whether miR-23a-3p in exosomes plays a role in HCC metastasis, we decreased the expression of miR-23a-3p. When the expression of miR-23a-3p in M2 macrophages was knocked down (Supporting Supplementary Fig. 2b), the expression of miR-23a-3p in M2 macrophage exosomes also decreased (Supporting Supplementary Fig. 2c). The upregulation of miR-23a-3p expression in HCC cells and HUVECs caused by M2 macrophage coculture was also inhibited (Supporting Supplementary Fig. 2d). When miR-23a-3p in exosomes was downregulated, the effect of M2 exosomes on HCC metastasis, angiogenesis, and vascular permeability was reduced, whereas an increase in miR-23a-3p expression restored the function of M2 macrophage exosomes (Fig. 4c-d and Supporting Supplementary Fig. 3). These findings indicate that M2 macrophage exosomes promote HCC metastasis by transferring miR-23a-3p.

\section{hnRNPA1 mediates miR-23a-3p packaging into exosomes.}

To study how miR-23a-3p is packaged into exosomes, we predicted the binding proteins of miR-23a-3p using the database of RBP specificities (RBPDB, http://rbpdb.ccbr.utoronto.ca/; threshold 0.6). Among the top three binding proteins, we focused on hnRNPA1 because the hnRNP family has been reported to participate in microRNA packaging into exosomes. When hnRNPA1 was knocked down in macrophages (Fig. 5a-b), the expression of miR-23a-3p in exosomes was also reduced (Fig. 5c). Moreover, the connection between miR-23a-3p and hnRNPA1 was confirmed by RIP assays (Fig. $5 d$ ). When cocultured with macrophages whose hnRNPA1 was knocked down, the upregulation of miR-23a-3p in HCC cells or HUVECs was also reversed (Fig. 5e). Subsequently, we investigated the effect of exosomes on HCC metastasis, angiogenesis, and vascular permeability after hnRNPA1 knockdown. The results show that the promotion of M2 exosomes on HCC metastasis, angiogenesis, and vascular permeability was weaker with hnRNPA1 knockdown (Fig. $5 f$ and Supporting Supplementary Fig. 5). These findings demonstrate that hnRNPA1-mediated miR-23a-3p sorting into exosomes promotes HCC metastasis, angiogenesis, and vascular permeability.

HCC stimulated by M2 exosomes recruits more macrophages. 
In the tumor microenvironment, inflammatory cells can regulate the progression of tumor cells, and tumor cells can also recruit inflammatory cells and other cells. Thus, we investigated the effect of HCC cells on macrophages. Fig. 6a-g shows that HCC cells could recruit macrophages as well as induce M2 macrophage polarization. Furthermore, HCC cells treated with M2 macrophage exosomes showed more M2 macrophage recruitment and polarization, as reflected by higher CD163 and IL-10 levels and lower TNF-a levels. In addition, we studied how HCC cells regulated macrophage phenotype. Chemokines are a class of small cytokines or secreted signaling proteins that have the ability to induce directional chemotaxis of nearby responding cells. The mobilization and infiltration of inflammatory monocytes mainly depends on the CCL2/CCR2 signaling pathway. CCL2 is secreted by hepatocytes or hepatic stellate cells and is involved in liver injury, hepatitis, and liver cancer. We found that HCC cells treated with M2 exosomes expressed higher levels of CCL2 (Fig. 6h). Then, we knocked down the expression of CCR2, the only known receptor for CCL2. The results showed that when the expression of CCR2 was reduced in macrophages (Fig. 6i), the effect of HCC cells on M2 macrophage recruitment and polarization was reduced (Fig. 6j-0). These findings indicate that $\mathrm{HCC}$ cells treated with M2 macrophage exosomes secrete more CCL2 and more significantly enhance M2 macrophage recruitment and polarization.

\section{PTEN and TJP1 are direct targets of miR-23a-3p}

TargetScan and miRDB were used to identify the targets of miR-23a-3p. Among the potential targets, we focused on PTEN and TJP1. To verify their association, luciferase reporter experiments were performed. The results showed that overexpressed miR-23a-3p reduced the luciferase activity of the wild-type 3'-UTR of PTEN and TJP1, but not the mutant (Supporting Fig. S5a-d). Overexpression of miR-23a-3p reduced the expression of PTEN and TJP1 in HCC cells and HUVECs at the mRNA and protein levels (Supporting Fig. S5e-h). Then, we further investigated the role of PTEN and TJP1 in HCC metastasis, angiogenesis, and vascular permeability. The HCC cells or HUVECs were transfected with PTEN and TJP1 before exosome incubation. The results showed that PTEN and TJP1 overexpression reduces the effect of M2 exosomes on HCC metastasis, EMT, angiogenesis, and vascular permeability (Fig. 7 and Supporting Supplementary Fig. 6). However, restoration of miR-23a-3p eliminated the effect of PTEN and TJP1 overexpression. These findings suggest that M2 exosomes with miR-23a-3p promote HCC metastasis, angiogenesis, and vascular permeability by targeting PTEN or TJP1.

\section{Discussion}

TME plays a vital role in the occurrence and development of tumors. Exosomes are involved in signal transmission between cells in the tumor microenvironment. Studies have shown that exosomes derived from TAMs or other immune cells can promote tumor metastasis, drug resistance, and immune escape. This study determined that M2 macrophage exosomes promote metastasis of HCC by transmitting miR23a-3p.

Tumor metastasis is a complex, multi-step process. First, tumor cells become more aggressive, and EMT is an important transition process for tumor cells to obtain high invasiveness. EMT is an important 
biological process in which epithelial cells are transformed into mesenchymal phenotype cells that play an important role in chronic inflammation, tissue remodeling, tumor metastasis, and fibrotic diseases ${ }^{18}$. Cells are characterized by interstitial cells, including disrupted cell polarity and basement membrane junction, enhanced migration and invasion, upregulated anti-apoptosis, and extracellular matrix degradation ${ }^{19,20}$. Studies have suggested that M2 exosomes promote tumor metastasis ${ }^{21-23}$. Whether M2 exosomes enhances EMT, particularly in HCC, requires further investigation. In our study, we found that M2 macrophage infiltration occurs with EMT in HCC. MiR-23a-3p was highly expressed in M2 macrophage-derived exosomes. MiR-23a-3p was transferred into HCC cells, which then exhibited reduced E-cadherin expression and increased N-cadherin and vimentin expression that promote HCC cell migration and invasion by targeting PTEN.

Tumor cells are separated from the primary tumor, invade the extracellular matrix, and inject blood vessels, then "seeds" enter the target organs and grow to form metastasis nodules. Therefore, angiogenesis and an increase in vascular permeability play important roles in the second stage of tumor metastasis ${ }^{24,25}$. The connection between vascular endothelial cells is essential to maintaining the integrity of vascular endothelial cells, including VE-cadherin, ZO-1, and claudin- $5^{26}$. This study shows that loss of these adhesion molecules weakens endothelial connections and increases vascular permeability, thereby destroying the blood vessel barrier and promoting cancer cell metastasis. These findings indicate that tumor-derived exosomes enhance vascular permeability. HCC cells transferred miR-103 into HUVECs, target junction proteins, increase vascular permeability, and promote HCC metastasis16. Hypoxia induces lung cancer cells to produce more exosomes. In addition, exosomes under hypoxic conditions contain more miR-23a that directly targets PHD1 and PHD2, leading to the increase of angiogenesis and vascular permeability ${ }^{27}$. However, whether M2 exosomes influence angiogenesis and vascular permeability in HCC remains unknown. In this study, we found that M2 macrophage infiltration occurred with angiogenesis in HCC. M2 exosomes with miR-23a-3p increased the expression of VEGFA, the proliferation and metastasis of HUVEC cells, and angiogenesis by targeting PTEN. Moreover, M2 exosomes with miR-23a-3p downregulated TJP1, occludin, and claudin 5, leading to increased vascular permeability.

Recently, the pre-metastatic niche concept has become a research topic of interest. The pre-metastatic niche provides a supportive microenvironment for disseminating tumor cells such as immunosuppression, abundant blood vessels, and inflammatory response ${ }^{28}$. Thus, the pre-metastatic niche has become a route for studying tumor metastasis. Studies have shown that exosomes participate in the formation of the pre-metastatic niche ${ }^{31}$. HCC cells with higher migration and invasion abilities secrete miR-1247-3p-rich exosomes that activate cancer-associated fibroblasts (CAFs), enhance HCC metastasis, invasion, and EMT via IL-6 and IL- $8^{32}$. However, how tumor cells stimulated by M2 exosomes recruit more M2 macrophages to provide a pre-metastatic niche for HCC metastasis remains unclear. In this study, we found that tumor cells stimulated by M2 exosomes secrete more CCL2 and induce macrophage infiltration and M2 polarization. CCL2/CCR2 is a classic monocyte/macrophage recruitment signaling pathway that plays an important role in HCC progression ${ }^{33-35}$. Blocking the CCL2/CCR2 signaling pathway can inhibit inflammatory cell infiltration and M2 polarization, thereby reversing the 
immunosuppressive state of the tumor microenvironment and activating the anti-tumor CD8 $+\mathrm{T}$ cell response ${ }^{36}$. After knocking down the receptor CCR2, the effect of the HCC cell supernatant on M2 macrophage infiltration was significantly reduced.

In conclusion, our study suggests that hnRNPA1-mediated packaging of miR-23a-3p into M2 macrophage exosomes promotes HCC cell metastasis by enhancing EMT, angiogenesis, and vascular permeability by targeting PTEN and TJP1. Moreover, HCC cells stimulated by M2-derived exosomes more extensively enhances M2 macrophage infiltration. Therefore, miR-23a-3p might serve as a potential therapeutic target for HCC metastasis.

\section{Conclusion}

In summary, in this study, we successfully constructed THP-1-induced M2 macrophage model and confirmed that it was associated with high infiltration, high metastasis rate and poor prognosis in HCC patients. We then extracted exosomes from M2 macrophages and carried out a series of functional experiments to verify its promoting effect on $\mathrm{HCC}$ cell metastasis and angiogenesis. The subsequent miRNAmicroarray helped us to determine miR-23a-3p, which was significantly elevated in M2 macrophage derived exosomes, and its high expression was closely related to hnRNPA1-mediated miR23a-3p packaging into exosomes. Luciferase reporter gene analysis confirmed that the targets of miR92a-3p were phosphatase and tensin homologue (PTEN) and tight junction protein 1 (TJP1). Then, we cocultured the exosomes derived from M2 macrophages with hepatocellular carcinoma cells. The results showed that HCC cells secreted more GM-CSF, VEGF, G-CSF, MCP-1 and IL-4, and then recruited more M2 macrophages.

\section{Abbreviations}

HCC: Hepatocellular carcinoma; TAMs: Tumor-associated macrophages; HUVEC: Human umbilical vein endothelial cell; MiRNA: MicroRNA; EMT: Epithelial-mesenchymal transition; PTEN: Phosphatase and tensin homolog; TJP1: Tight junction protein 1; PMA, phorbol-12-myristate-13 acetate; TEM: Transmission electron microscopy; PCR: Polymerase chain reaction; RIP: RNA immunoprecipitation; PMSF:

Phenylmethylsulfonyl fluoride; GSEA: Gene Set Enrichment Analysis; CAFs: Cancer-associated fibroblasts

\section{Declarations}

\section{Acknowledgements}

The study was supported by the National Natural Science Foundation of China (81972768).

\section{Author contributions}

GY.H ,Y.Z,QY.W and L.Z\Z.L analyzed and/or interpreted the data. YW.L performed the experiments. ZQ.C provided study supervision. All authors have read and approved the final version. 


\section{Funding}

This work was supported by research grants from the National Natural Science Foundation of China (81972768, 81270553, 81300363, 81521004 and 81572262), Young and middle-aged academic leaders of Jiangsu University Blue Project 2019, Support plan 2019 for excellent young and middle-aged teachers of Nanjing Medical University, the Fund of State Key Laboratory of Reproductive Medicine, Nanjing Medical University (SKLRM-K201706), Jiangsu Youth Medical Talents (QNRC2016580), the Outstanding Young scholar Foundation of Jiangsu Province, China (BK20170106), the Open Fund of State Key Laboratory of Pharmaceutical Biotechnology, Nan-jing University, China (KF-GN-201903), the Southeast University and Nanjing Medical University Cooperative Research Project, China (2019DN0003), Jiangsu Province's Key provincial Talents Program (ZDRCA2016028), 333 high class "Talented Man Project" (BRA2016516), and Major Program of Science and technology innovation fund of Nanjing Medical University (2017NJMUCX005).

\section{Availability of data and materials}

All data in this study are available upon request.

\section{Ethical Approval and Consent to participate}

This research has got the permission by the ethics committee of The First Affiliated Hospital of Nanjing Medical University.

\section{Consent for publication}

All authors agree to the content of the paper.

\section{Competing interests}

The authors have no competing interests to declare.

\section{References}

1. Maluccio M, Covey A. Recent progress in understanding, diagnosing, and treating hepatocellular carcinoma. CA Cancer J Clin. 2012;62:394-9. doi:10.3322/caac.21161.

2. Ferlay $\mathrm{J}$, et al. Cancer incidence and mortality worldwide: sources, methods and major patterns in GLOBOCAN 2012. Int J Cancer. 2015;136:E359-86. doi:10.1002/ijc.29210.

3. Davidson RS, Nwogu CE, Brentjens MJ, Anderson TM. The surgical management of pulmonary metastasis: current concepts. Surg Oncol. 2001;10:35-42. doi:10.1016/s0960-7404(01)00013-5.

4. Zhang SM, et al. Prognostic analysis of pulmonary metastases from hepatocellular carcinoma. Hepatol Int. 2008;2:237-43. doi:10.1007/s12072-008-9052-7. 
5. Albini A, Sporn MB. The tumour microenvironment as a target for chemoprevention. Nat Rev Cancer. 2007;7:139-47. doi:10.1038/nrc2067.

6. Hu M, Polyak K. Microenvironmental regulation of cancer development. Curr Opin Genet Dev. 2008;18:27-34. doi:10.1016/j.gde.2007.12.006.

7. Jaynes JM, et al. Mannose receptor (CD206) activation in tumor-associated macrophages enhances adaptive and innate antitumor immune responses. Sci Transl Med 12, doi:10.1126/scitransImed.aax6337 (2020).

8. Li M, et al. Remodeling tumor immune microenvironment via targeted blockade of PI3K-y and CSF$1 /$ CSF-1R pathways in tumor associated macrophages for pancreatic cancer therapy. $\mathrm{J}$ Control Release. 2020;321:23-35. doi:10.1016/j.jconrel.2020.02.011.

9. Sami E, Paul BT, Koziol JA, EIShamy WM. The Immunosuppressive Microenvironment in BRCA1-IRISOverexpressing TNBC Tumors Is Induced by Bidirectional Interaction with Tumor-Associated Macrophages. Cancer Res. 2020;80:1102-17. doi:10.1158/0008-5472.Can-19-2374.

10. Qian BZ, Pollard. J. W. Macrophage diversity enhances tumor progression and metastasis. Cell. 141, 39-51, doi:10.1016/j.cell.2010.03.014 (2010).

11. Shirabe K, et al. Role of tumor-associated macrophages in the progression of hepatocellular carcinoma. Surg Today. 2012;42:1-7. doi:10.1007/s00595-011-0058-8.

12. Fan $Q M$, et al. Tumor-associated macrophages promote cancer stem cell-like properties via transforming growth factor-beta1-induced epithelial-mesenchymal transition in hepatocellular carcinoma. Cancer Lett. 2014;352:160-8. doi:10.1016/j.canlet.2014.05.008.

13. Théry C, Zitvogel L, Amigorena S. Exosomes: composition, biogenesis and function. Nat Rev Immunol. 2002;2:569-79. doi:10.1038/nri855.

14. Wang X, et al. Hypoxic Tumor-Derived Exosomal miR-301a Mediates M2 Macrophage Polarization via PTEN/PI3Ky to Promote Pancreatic Cancer Metastasis. Cancer Res. 2018;78:4586-98. doi:10.1158/0008-5472.Can-17-3841.

15. Tian XP, et al Acidic Microenvironment Up-Regulates Exosomal miR-21 and miR-10b in Early-Stage Hepatocellular Carcinoma to Promote Cancer Cell Proliferation and Metastasis. Theranostics. 9, 1965-1979, doi:10.7150/thno.30958 (2019).

16. Fang $\mathrm{JH}$, et al. Hepatoma cell-secreted exosomal microRNA-103 increases vascular permeability and promotes metastasis by targeting junction proteins. Hepatology. 2018;68:1459-75. doi:10.1002/hep.29920.

17. Greenburg G, Hay ED. Epithelia suspended in collagen gels can lose polarity and express characteristics of migrating mesenchymal cells. J Cell Biol. 1982;95:333-9. doi:10.1083/jcb.95.1.333.

18. Shibue T, Brooks MW, Weinberg RA. An integrin-linked machinery of cytoskeletal regulation that enables experimental tumor initiation and metastatic colonization. Cancer Cell. 2013;24:481-98. doi:10.1016/j.ccr.2013.08.012. 
19. Jin H, et al. Snail is critical for tumor growth and metastasis of ovarian carcinoma. Int J Cancer. 2010;126:2102-11. doi:10.1002/ijc.24901.

20. Cheng H, Wang Z, Fu L, Xu T. Macrophage Polarization in the Development and Progression of Ovarian Cancers: An Overview. Front Oncol. 2019;9:421. doi:10.3389/fonc.2019.00421.

21. Lan J, et al. M2 Macrophage-Derived Exosomes Promote Cell Migration and Invasion in Colon Cancer. Cancer Res. 2019;79:146-58. doi:10.1158/0008-5472.Can-18-0014.

22. Wang F, et al. Tumor-derived exosomes induce PD1(+) macrophage population in human gastric cancer that promotes disease progression. Oncogenesis 7, 41, doi:10.1038/s41389-018-0049-3 (2018).

23. Liu Y, Cao X. Characteristics and Significance of the Pre-metastatic Niche. Cancer Cell. 30., 668-681, doi:10.1016/j.ccell.2016.09.011 (2016).

24. Ergun S, Tilki D, Oliveira-Ferrer L, Schuch G, Kilic N. Significance of vascular stabilization for tumor growth and metastasis. Cancer Lett. 2006;238:180-7. doi:10.1016/j.canlet.2005.06.033.

25. Bazzoni G, Dejana E. Endothelial cell-to-cell junctions: molecular organization and role in vascular homeostasis. Physiol Rev. 2004;84:869-901. doi:10.1152/physrev.00035.2003.

26. Hsu YL, et al. Hypoxic lung cancer-secreted exosomal miR-23a increased angiogenesis and vascular permeability by targeting prolyl hydroxylase and tight junction protein ZO-1. Oncogene. 2017;36:4929-42. doi:10.1038/onc.2017.105.

27. Feng W, Dean DC, Hornicek FJ, Shi H, Duan Z. Exosomes promote pre-metastatic niche formation in ovarian cancer. Mol Cancer. 2019;18:124. doi:10.1186/s12943-019-1049-4.

28. Guo Y, et al. Effects of exosomes on pre-metastatic niche formation in tumors. Mol Cancer. 18., 39, doi:10.1186/s12943-019-0995-1 (2019).

29. Plebanek MP, et al. Pre-metastatic cancer exosomes induce immune surveillance by patrolling monocytes at the metastatic niche. Nat Commun. 2017;8:1319. doi:10.1038/s41467-017-01433-3.

30. Zeng Z, et al. Cancer-derived exosomal miR-25-3p promotes pre-metastatic niche formation by inducing vascular permeability and angiogenesis. Nat Commun. 2018;9:5395. doi:10.1038/s41467018-07810-w.

31. Fang $T$, et al. Tumor-derived exosomal miR-1247-3p induces cancer-associated fibroblast activation to foster lung metastasis of liver cancer. Nat Commun. 2018;9:191. doi:10.1038/s41467-017-025830 .

32. Wang $D$, et al. APOBEC3B interaction with PRC2 modulates microenvironment to promote HCC progression. Gut. 2019;68:1846-57. doi:10.1136/gutjnl-2018-317601.

33. Eggert $T$, et al. Distinct Functions of Senescence-Associated Immune Responses in Liver Tumor Surveillance and Tumor Progression. Cancer Cell 30, 533-547. doi:10.1016/j.ccell.2016.09.003 (2016).

34. Zhou SL, et al. Tumor-Associated Neutrophils Recruit Macrophages and T-Regulatory Cells to Promote Progression of Hepatocellular Carcinoma and Resistance to Sorafenib. Gastroenterology. 
2016;150:1646-58.e1617. doi:10.1053/j.gastro.2016.02.040.

35. Li X, et al. Targeting of tumour-infiltrating macrophages via CCL2/CCR2 signalling as a therapeutic strategy against hepatocellular carcinoma. Gut. 2017;66:157-67. doi:10.1136/gutjnl-2015-310514.

36. Petty AJ, et al. Hedgehog signaling promotes tumor-associated macrophage polarization to suppress intratumoral CD8+ T cell recruitment. J Clin Invest. 2019;129:5151-62. doi:10.1172/jci128644.

\section{Figures}

\section{Figure 1}

TAMs infiltration is correlated with metastasis and poor prognosis in HCC. a: Immunohistochemistry of M2 macrophage marker CD163 in tumor tissues from HCC patients with or without metastasis. b: Overall survival of CD163-positive macrophages in HCC patients. C: GSEA was performed using TCGA HCC datasets to test the relationship between the expression of CCL2 in HCC tissues and EMT and angiogenesis.

\section{Figure 2}

M2 macrophage exosomes promote HCC cell metastasis in vitro. a: Transmission electron micrograph of exosomes in M2-polarized macrophages. b: Exosome size distribution as analyzed by Nanosight particle tracking. c: Western blot analysis of exosome marker CD63, CD81, and CD9 expression. d: Exosome internalization by HCC cells was assessed using PKH67. e: The effect of M2 macrophage exosomes on $\mathrm{HCC}$ cell metastasis as assessed using the Transwell $\circledast$ assay. $\mathrm{f}$ : The effect of $\mathrm{M} 2$ macrophage exosomes on HCC cell EMT was assessed by western blotting. ${ }^{*} P<0.05,{ }^{*} P<0.01,{ }^{\star * *} P<0.001$.

\section{Figure 3}

M2 macrophage exosomes promote angiogenesis and increase vascular permeability. a: Exosomes internalization by HUVEC cells as assessed by PKH67. b: The effect of M2 macrophage exosomes on the release of VEGFA as assessed by ELISA. c, d: The effect of M2 macrophage exosomes on the proliferation and migration of HUVECs as evaluated using the CCK-8 and Transwell ${ }^{\circledR}$ assays. e: The effect of M2 macrophage exosomes on angiogenesis as evaluated using a tube formation assay. $\mathrm{f}, \mathrm{g}$ : The effect of M2 macrophage exosomes on vascular permeability as tested by transendothelial invasion and endothelial permeability. h: The effect of M2 macrophage exosomes on the expression of vascular adhesion molecules as assessed by western blotting. ${ }^{*} P<0.05,{ }^{\star *} P<0.01$. 


\section{Figure 4}

M2 macrophage exosomes transferred miR-23a-3p into HCC cells and HUVECs. a: The expression of miR23a-3p in M0 and M2 exosomes as tested by RT-PCR. b: Cy3-miR-23a-3p internalization by HCC cells and HUVECs was evaluated by fluorescence microscopy. c: The effect of M2 macrophage exosomal miR-23a$3 p$ on HCC cell metastasis as assessed by the Transwell@ assay. d: The effect of M2 macrophage exosomal miR-23a-3p on HCC cell EMT as evaluated by western blotting. ${ }^{*} P<0.05,{ }^{\star *} \mathrm{P}<0.01$.

\section{Figure 5}

HnRNPA1 mediated miR-23a-3p packaging into M2 macrophage-derived exosomes. a, b: Knockdown of hnRNPA1 was assessed by qRT-PCR and western blotting. c: The effect of hnRNPA1 knock down of the expression of miR-23a-3p in M2 macrophage exosomes as analyzed by RT-PCR. $d$ : The combination of miR-23a-3p and hnRNPA1 was evaluated by the RIP assay. e: The expression of miR-23a-3p in HCC cells and HUVECs when cocultured with M2 macrophages after hnRNPA1 knock down and assessed by RTPCR. f: The effect of M2 macrophage exosomes after hnRNPA1 knock down on HCC cell metastasis and evaluated by a Transwell ${ }^{\circledR}$ assay. ${ }^{*} \mathrm{P}<0.05,{ }^{\star *} \mathrm{P}<0.01,{ }^{*} * \mathrm{P}<0.001$.

\section{Figure 6}

HCC cells induce macrophage recruitment and polarization. $a$, b: Macrophage recruitment induced by HCC cells was assessed by a Transwell ${ }^{\circledR}$ assay. C: The expression of M2 macrophage marker CD163 in macrophages induced by HCC cells as tested by qRT-PCR. $\mathrm{d}-\mathrm{g}$ : The expression of IL-10 and TNF-ain macrophages induced by HCC cells as assessed by qRT-PCR and ELISA. h: The effect of M2 macrophage exosomes on the secretion of CCL2 as evaluated by qRT-PCR and ELISA. i: Knock down of CCR2 as analyzed by qRT-PCR and western blotting. j, $k$ : The effect of CCR2 knock down on macrophage recruitment as induced by HCC cells and evaluated by a Transwell $\circledast$ assay. I: The effect of CCR2 knock down on the expression of M2 macrophage marker CD163 in macrophages induced by HCC cells as assessed by qRT-PCR. $\mathrm{m}-0$ : The effect of CCR2 knock down on the expression of IL-10 and TNF-ain macrophages induced by HCC cells and tested by qRT-PCR and ELISA. ${ }^{*} P<0.05,{ }^{\star *} \mathrm{P}<0.01,{ }^{\star \star \star} \mathrm{P}<0.001$.

\section{Figure 7}

M2 macrophage exosomal miR-23a-3p promotes HCC cell metastasis and EMT by targeting PTEN. a: The effect of PTEN on the metastasis of HCC cells induced by M2 macrophage exosomal miR-23a-3p and 
tested by a Transwell $\circledast$ assay. b: The effect of PTEN on the EMT of HCC cells induced by M2 macrophage exosomal miR-23a-3p and tested by western blotting. ${ }^{\star} \mathrm{P}<0.05,{ }^{\star *} \mathrm{P}<0.01$.

\section{Supplementary Files}

This is a list of supplementary files associated with this preprint. Click to download.

- supplementaryinformationfiles.pdf 